Proceedings of the Edinburgh Mathematical Society (2006) 49, 637-656 (C)

DOI:10.1017/S0013091505000532 Printed in the United Kingdom

\title{
NONLINEAR NON-LOCAL BOUNDARY-VALUE PROBLEMS AND PERTURBED HAMMERSTEIN INTEGRAL EQUATIONS
}

\author{
GENNARO INFANTE ${ }^{1}$ AND J. R. L. WEBB ${ }^{2}$ \\ ${ }^{1}$ Dipartimento di Matematica, Università della Calabria, \\ 87036 Arcavacata di Rende, Cosenza, Italy (g.infante@unical.it) \\ ${ }^{2}$ Department of Mathematics, University of Glasgow, \\ Glasgow G12 8QW, UK (jrlw@maths.gla.ac.uk)
}

(Received 14 April 2005)

Abstract Motivated by some non-local boundary-value problems (BVPs) that arise in heat-flow problems, we establish new results for the existence of non-zero solutions of integral equations of the form

$$
u(t)=\gamma(t) \alpha[u]+\int_{G} k(t, s) f(s, u(s)) \mathrm{d} s,
$$

where $G$ is a compact set in $\mathbb{R}^{n}$. Here $\alpha[u]$ is a positive functional and $f$ is positive, while $k$ and $\gamma$ may change sign, so positive solutions need not exist. We prove the existence of multiple non-zero solutions of the BVPs under suitable conditions. We show that solutions of the BVPs lose positivity as a parameter decreases. For a certain parameter range not all solutions can be positive, but for one of the boundary conditions we consider we show that there are positive solutions for certain types of nonlinearity. We also prove a uniqueness result.

Keywords: fixed-point index; cone; positive solution

2000 Mathematics subject classification: Primary 34B18

Secondary 34B10; 47H10; 47H30

\section{Introduction}

We discuss nonlinear non-local boundary-value problems (BVPs) for equations of the form

$$
-u^{\prime \prime}(t)=f(t, u(t)), \quad t \in(0,1),
$$

where $f$ is a non-negative function, with one of the two non-local boundary conditions (BCs)

$$
\begin{aligned}
& u^{\prime}(0)+\alpha[u]=0, \quad \beta u^{\prime}(1)+u(\eta)=0, \quad \eta \in[0,1], \\
& u(0)=\alpha[u], \quad \beta u^{\prime}(1)+u(\eta)=0, \quad \eta \in[0,1] .
\end{aligned}
$$

We are particularly interested in positivity properties of non-trivial solutions.

Both BVPs are handled here by studying the same type of perturbed Hammerstein integral equation. 
As in $[4,9]$, these BVPs arise in the study of the steady states of a heated bar with a thermostat, where a controller at $t=1$ adds or removes heat according to the temperature detected by a sensor at $t=\eta$. A special case of the BC (1.3) also occurs in studying the membrane response of a spherical cap $[\mathbf{1}, \mathbf{3}, \mathbf{1 7}, \mathbf{1 8}]$.

We shall take $\alpha[u]$ to be a non-negative affine functional given by

$$
\alpha[u]=A_{0}+\int_{0}^{1} u(s) \mathrm{d} A(s),
$$

involving a Stieltjes integral, which includes the particularly natural BCs

$$
u^{\prime}(0)+\alpha u(0)=0 \quad \text { or } \quad u(0)=U
$$

which, in the heated bar case, correspond, respectively, to natural heat loss at $t=0$ (or another controller at $t=0$ ) or to the left-hand end of the bar being maintained at a fixed temperature. It also includes multi-point problems when $\alpha[u]=\sum_{i=1}^{m} \alpha_{i} u\left(\xi_{i}\right)$ and a continuously distributed case when $\alpha[u]=\int_{0}^{1} \alpha(s) u(s) \mathrm{d} s$.

One motivation is that the linear problem

$$
-u^{\prime \prime}(t)=y(t)
$$

with BCs

$$
u^{\prime}(0)=0, \quad \beta u^{\prime}(1)+u(0)=0,
$$

which is a special case of BC (1.2) with $\eta=0$, has been studied by Guidotti and Merino [4] when $\beta>0$ (their version is equivalent to ours via a change of variable). They were interested in loss of positivity as $\beta$ decreases towards 0 and showed that (in our notation) the solution $u$ of (1.5), (1.6) is positive for every $y \geqslant 0$ if and only if $\beta \geqslant 1$. However, if $\beta \geqslant \frac{1}{2}$ and the symmetric and antisymmetric parts of $y$ (relative to the midpoint) are both positive, then $u$ is again positive.

We allow the sensor to be placed at an arbitrary point $\eta \in[0,1]$ and we exhibit loss of positivity for the nonlinear problems by showing the existence of non-zero solutions that may change sign, and the subinterval of $[0,1]$ on which they are positive decreases in size as $\beta$ decreases.

We adopt the view that the parameter $\beta$ is under our control, while the other parameters are fixed, and we shall discuss all possible ranges of values of $\beta$.

In particular we show that, for $\beta \geqslant 1-\eta$, positive solutions exist under suitable conditions on $f$ (such as sublinearity or superlinearity). But, in contrast to the linear case, multiple positive solutions exist when $f$ has a suitable oscillatory behaviour.

When $0<\beta<1-\eta$ there cannot exist positive solutions for all positive right-hand sides, but we show that there are (multiple) non-zero solutions that are positive on the interval $[0, b]$ for any $b$ with $\eta<b<\beta+\eta<1$. This shows, by the shrinking of the interval $[0, b]$, the loss of positivity as $\beta$ decreases. 
For $\beta<0$ and $\beta+\eta>0$ we show that there are solutions that are negative on an interval $[a, 1]$ for $\beta+\eta<a<1$.

For $\beta<0$ and $\beta+\eta<0$ for $\mathrm{BC}(1.2)$, there are solutions that are negative on $[0,1]$ but, for $\mathrm{BC}(1.3)$, it turns out that there again exist positive solutions; this is because $\beta+\eta=0$ is a singular case for this BVP.

In [9] we showed a regain-of-positivity result for the BC $u^{\prime}(0)=0$ when, for example, $f(t, u)=f(u)$ is decreasing and bounded. If $\beta$ satisfies

$$
\frac{1}{2}\left(1-\gamma \eta^{2}\right) \leqslant \beta<1-\eta
$$

with $\gamma=f_{\min } / f_{\max }$, then a solution exists that is actually positive on all of $[0,1]$. In the present paper we extend this to a special case of $\mathrm{BC}(1.2)$ but we have no such result for $\mathrm{BC}(1.3)$.

We also prove some uniqueness results and show, by elementary arguments, that if $f(t, u)$ is decreasing in $u$, then solutions of (1.1) and (1.2), and (1.1)-(1.3), are unique if $\alpha[u]$ is constant.

The problems we discuss include examples of so-called three-point and multi-point BVPs, and we treat these in a unified way. Several such non-local problems have been extensively studied recently (see, for example, $[\mathbf{6}, \mathbf{8}, \mathbf{1 2}, \mathbf{1 5}, \mathbf{1 9}]$ and the papers cited therein). In particular, Karakostas and Tsamatos [10] study BCs given by RiemannStieltjes integrals.

The methodology used to treat such problems has been to write the BVP as a Hammerstein integral equation,

$$
u(t)=\int_{0}^{1} \tilde{k}(t, s) f(s, u(s)) \mathrm{d} s:=S u(t)
$$

and find a solution as a fixed point of the operator $S$ by means of the theory of fixed-point index for compact mappings.

We also use fixed-point-index theory on a suitable cone $K$ in $C[0,1]$, but our new approach is to use an equivalent equation of the form

$$
u(u)=\gamma(t) \alpha[u]+\int_{0}^{1} k(t, s) f(s, u(s)) \mathrm{d} s:=A u(t)+F u(t) .
$$

Here $\gamma(t)=\beta+\eta-t$ for $\mathrm{BC}(1.2)$, which changes sign on the interval $[0,1]$ when $0<\beta+\eta<1$, and the kernel also changes sign. We suppose that $\alpha[u] \geqslant 0$ for $u \in K$.

The advantage of our method is that we deal with a simpler kernel in (1.8) than we would have to deal with using (1.7), at the cost of having the perturbation. We develop some new theory to deal with this type of perturbed Hammerstein integral equation in a more general setting, with $[0,1]$ replaced by a compact subset $G$ of $\mathbb{R}^{n}$. We use our knowledge from [7], which treated integral equations with sign-changing kernels, to deal with this perturbed situation and obtain new results for the fixed-point index on various sets that exploit the decomposition. 


\section{Existence of non-trivial solutions of perturbed Hammerstein integral equations}

We study fixed points of the operator

$$
u(t)=\gamma(t) \alpha[u]+\int_{G} k(t, s) f(s, u(s)) \mathrm{d} s:=T u(t),
$$

where $G$ is a compact set of positive measure in $\mathbb{R}^{n}$. We set

$$
A u(t):=\gamma(t) \alpha[u] \text { and } F u(t):=\int_{G} k(t, s) f(s, u(s)) \mathrm{d} s,
$$

so that $T=A+F$.

We work in the space $C(G)$ of continuous functions endowed with the usual supremum norm, and shall use the well-known classical fixed-point index for compact maps. We recall some facts here and refer the reader to $[\mathbf{2}]$ or $[\mathbf{5}]$ for further information.

Let $K$ be a cone in a Banach space $X$, that is, $K$ is a closed convex set such that $\lambda x \in K$ for $x \in K$ and $\lambda \geqslant 0$ and $K \cap(-K)=0$.

If $\Omega$ is a bounded open subset of $K$ (in the relative topology), we denote by $\bar{\Omega}$ and $\partial \Omega$ the closure and the boundary relative to $K$. When $D$ is an open bounded subset of $X$ we write $D_{K}=D \cap K$, an open subset of $K$. The following result is well known in fixed-point-index theory (see, for example, $[\mathbf{2}, \mathbf{5}]$ ).

Lemma 2.1. Let $D$ be an open bounded set with $D_{K} \neq \emptyset$ and $\bar{D}_{K} \neq K$. Assume that $T: \bar{D}_{K} \rightarrow K$ is a compact map such that $x \neq T x$ for $x \in \partial D_{K}$. The fixed-point index $i_{K}\left(T, D_{K}\right)$ then has the following properties.

(i) If there exists $e \in K \backslash\{0\}$ such that $x \neq T x+\lambda e$ for all $x \in \partial D_{K}$ and all $\lambda>0$, then $i_{K}\left(T, D_{K}\right)=0$.

(ii) If $0 \in D_{K}$ and $T x \neq \lambda x$ for all $x \in \partial D_{K}$ and all $\lambda>1$, then $i_{K}\left(T, D_{K}\right)=1$.

For example, (ii) holds if $\|T x\| \leqslant\|x\|$ for $x \in \partial D_{K}$.

We now make assumptions on $f, \alpha, \gamma$ and the kernel $k$, which will enable us to define a suitable cone and show that our integral operator is compact.

Recall that $f$ is said to satisfy the Carathéodory conditions if, for each $u, s \mapsto f(s, u)$ is measurable and, for almost every $s, u \mapsto f(s, u)$ is continuous.

$\left(\mathrm{C}_{1}\right)$ Suppose that, for every $r>0, f: G \times[-r, r] \rightarrow[0, \infty)$ satisfies Carathéodory conditions and there exists a measurable function $g_{r}: G \rightarrow[0, \infty)$ such that

$$
f(s, u) \leqslant g_{r}(s) \text { for almost all } s \in G \text { and all } u \in[-r, r] .
$$

$\left(\mathrm{C}_{2}\right) k: G \times G \rightarrow \mathbb{R}$ is measurable, and for every $\tau \in G$ we have

$$
\lim _{t \rightarrow \tau} \int_{G}|k(t, s)-k(\tau, s)| g_{r}(s) \mathrm{d} s=0 .
$$


$\left(\mathrm{C}_{3}\right)$ There exist a closed subset $G_{0} \subset G$ with meas $\left(G_{0}\right)>0$, a measurable function $\Phi: G \rightarrow[0, \infty)$ and a constant $c_{1} \in(0,1]$ such that

$$
\begin{aligned}
|k(t, s)| \leqslant \Phi(s) & \text { for } t \in G \text { and almost every } s \in G, \\
k(t, s) \geqslant c_{1} \Phi(s) & \text { for } t \in G_{0} \text { and almost every } s \in G .
\end{aligned}
$$

$\left(\mathrm{C}_{4}\right)$ For each $r>0$ there exists an $M_{r}<\infty$ such that

$$
\int_{G} \Phi(s) g_{r}(s) \mathrm{d} s \leqslant M_{r} .
$$

$\left(\mathrm{C}_{5}\right) \gamma: G \rightarrow \mathbb{R}$ is continuous and there exists a constant $c_{2} \in(0,1]$ such that

$$
\gamma(t) \geqslant c_{2}\|\gamma\| \text { for } t \in G_{0} .
$$

$\left(\mathrm{C}_{6}\right) \alpha: K \rightarrow \mathbb{R}^{+}$is a continuous functional with

$$
\alpha[u]=A_{0}+\int_{G} u(s) \mathrm{d} A(s),
$$

where $\mathrm{d} A$ is a Lebesgue-Stieltjes measure with $A_{1}:=\int_{G} \mathrm{~d} A(s)<\infty$.

$\left(\mathrm{C}_{7}\right)$ The function $t \mapsto k(t, s)$ is integrable with respect to the measure $\mathrm{d} A$, that is

$$
\mathcal{K}(s):=\int_{G} k(t, s) \mathrm{d} A(t)
$$

is well defined.

Under these hypotheses we can and shall work in the following cone

$$
K=\left\{u \in C(G): \min \left\{u(t): t \in G_{0}\right\} \geqslant c\|u\|\right\}, \quad \text { where } c=\min \left\{c_{1}, c_{2}\right\} .
$$

This type of cone was introduced by the authors in [8] and later used in [7]. This is similar to, but larger than, the cone of non-negative functions used in $[\mathbf{1 2}, \mathbf{1 4}]$. The latter type of cone goes back to Krasnosel'skiı̌ [11], and Guo [5]. Note that functions in $K$ are positive on the subset $G_{0}$ but may change sign on $G$. Henceforth, in this paper, $K$ will denote the cone in (2.2).

Definition 2.2. We write $K_{r}=\{u \in K:\|u\|<r\}, \bar{K}_{r}=\{u \in K:\|u\| \leqslant r\}$, and define

$$
\Gamma=\int_{G} \gamma(t) \mathrm{d} A(t)
$$

We first show that $T: K \rightarrow K$ is compact, that is, $T$ is continuous and $\overline{T(Q)}$ is compact for each bounded subset $Q \subset K$.

Theorem 2.3. Assume that $\left(C_{1}\right)-\left(C_{6}\right)$ hold for some $r>0$. Then $T$ maps $\bar{K}_{r}$ into $K$ and is compact. When these hypotheses hold for each $r>0, T$ is compact and maps $K$ into $K$. 
Proof. The compactness of $F$ follows from [16, Proposition 3.1, Chapter 5]. $A$ is compact since it maps a bounded set into a bounded subset of a one-dimensional space and thus $T=A+F$ is compact. To see that $T: \bar{K}_{r} \rightarrow K$, for $u \in \bar{K}_{r}$ and $t \in G$, we have

$$
|T u(t)| \leqslant|\gamma(t)| \alpha[u]+\int_{G}|k(t, s)| f(s, u(s)) \mathrm{d} s
$$

so that

$$
\|T u\| \leqslant\|\gamma\| \alpha[u]+\int_{G} \Phi(s) f(s, u(s)) \mathrm{d} s .
$$

Also,

$$
\min _{t \in G_{0}}\{T u(t)\} \geqslant c_{2}\|\gamma\| \alpha[u]+c_{1} \int_{G} \Phi(s) f(s, u(s)) \mathrm{d} s \geqslant c\left[\|\gamma\| \alpha[u]+\int_{G} \Phi(s) f(s, u(s)) \mathrm{d} s\right],
$$

where $c=\min \left\{c_{1}, c_{2}\right\}$. Hence, $T u \in K$ for every $u \in \bar{K}_{r}$.

Let $q: C(G) \rightarrow \mathbb{R}$ denote the continuous function $q(u)=\min \left\{u(t): t \in G_{0}\right\}$. We shall use the open set $V_{\rho}=\{u \in K: q(u)<\rho\}$. $V_{\rho}$ is equal to the set called $\Omega_{\rho / c}$ in [7], but we believe the notation $V_{\rho}$ is more natural and also makes it clear that choosing $c$ as large as possible yields a weaker condition to be satisfied by $f$ in Lemma 2.4.

We first prove a lemma which implies that the index is zero. This is new because we exploit the fact that, in some cases, $\alpha[u]$ is bounded below by a positive constant rather than being merely non-negative.

Lemma 2.4. Assume that there exists $\rho>0$ such that $u \neq T u$ for $u \in \partial V_{\rho}$ and that the following condition applies.

$\left(\mathrm{I}_{\rho}^{0}\right)$ There exists a measurable function $\psi_{\rho}: G_{0} \rightarrow \mathbb{R}_{+}$such that

$$
\begin{array}{cl}
f(s, u) \geqslant \rho \psi_{\rho}(s) & \text { for all } u \in[\rho, \rho / c] \text { and almost all } s \in G_{0}, \\
\alpha[u] \geqslant \alpha_{0} \rho & \text { for } u \in \partial V_{\rho}, \alpha_{0} \geqslant 0,
\end{array}
$$

and

$$
c_{2}\|\gamma\| \alpha_{0}+\inf _{t \in G_{0}} \int_{G_{0}} k(t, s) \psi_{\rho}(s) \mathrm{d} s \geqslant 1
$$

We then have $i_{K}\left(T, V_{\rho}\right)=0$.

Proof. Let $e(t) \equiv 1$ for $t \in G$. Then $e \in K$. We prove that

$$
u \neq T u+\lambda e \text { for } u \in \partial V_{\rho} \text { and } \lambda>0 .
$$

In fact, if this is not the case, there exist $u \in \partial V_{\rho}$ and $\lambda>0$ such that $u=T u+\lambda e$. We then have, for $t \in G_{0}$,

$$
\begin{aligned}
u(t) & =\gamma(t) \alpha[u]+\int_{G} k(t, s) f(s, u(s)) \mathrm{d} s+\lambda \\
& \geqslant c_{2}\|\gamma\| \alpha_{0} \rho+\rho \int_{G_{0}} k(t, s) \psi_{\rho}(s) \mathrm{d} s+\lambda .
\end{aligned}
$$


By $\left(\mathrm{I}_{\rho}^{0}\right)$, this implies that $q(u) \geqslant \rho+\lambda>\rho$, contradicting $u \in \partial V_{\rho}$. Hence, Lemma 2.1(i) gives $i_{K}\left(T, V_{\rho}\right)=0$.

Remark 2.5. When $G=[0,1], G_{0}=[a, b], f(t, u)=g(t) h(u)$, where $\Phi g \in L^{1}$ and $h$ is continuous, we have

$$
\psi_{\rho}(s)=g(s) h_{\rho, \rho / c}, \quad \text { where } h_{\rho, \rho / c}=\inf \{h(u) / \rho: \rho \leqslant u \leqslant \rho / c\} .
$$

Then (2.3) reads more simply:

$$
c_{2}\|\gamma\| \alpha_{0}+h_{\rho, \rho / c} \frac{1}{M} \geqslant 1
$$

where

$$
\frac{1}{M}=\inf _{t \in[a, b]} \int_{a}^{b} k(t, s) g(s) \mathrm{d} s .
$$

This shows that Lemma 2.4 is an extension of results in $[\mathbf{7}, \mathbf{8}]$.

We next prove a result that implies that the index is 1 .

Lemma 2.6. Suppose $\Gamma<1$ and assume that there exists $\rho>0$ such that $u \neq T u$ for $u \in \partial K_{\rho}$ and

$\left(\mathrm{I}_{\rho}^{1}\right)$ there exists a measurable function $\phi_{\rho}: G \rightarrow \mathbb{R}_{+}$such that $\mathcal{K} \phi_{\rho} \in L^{1}(G)$,

$$
f(s, u) \leqslant \rho \phi_{\rho}(s) \text { for all } u \in[-\rho, \rho] \text { and almost all } s \in G
$$

and

$$
\frac{A_{0}\|\gamma\|}{(1-\Gamma) \rho}+\frac{\|\gamma\|}{(1-\Gamma)} \int_{G} \mathcal{K}(s) \phi_{\rho}(s) \mathrm{d} s+\sup _{t \in G} \int_{G}|k(t, s)| \phi_{\rho}(s) \mathrm{d} s \leqslant 1 .
$$

We then have $i_{K}\left(T, K_{\rho}\right)=1$.

Proof. We show that $T u \neq \lambda u$ for every $u \in \partial K_{\rho}$ and for every $\lambda>1$. In fact, if there exists $\lambda>1$ and $u \in \partial K_{\rho}$ such that $T u=\lambda u$, then

$$
\lambda u(t)=\gamma(t) \alpha[u]+\int_{G} k(t, s) f(s, u(s)) \mathrm{d} s .
$$

Therefore, we have

$$
\lambda \int_{G} u(t) \mathrm{d} A(t)=\alpha[u] \Gamma+\int_{G} \mathcal{K}(s) f(s, u(s)) \mathrm{d} s .
$$

Hence,

$$
(\lambda-\Gamma) \alpha[u]=\lambda A_{0}+\int_{G} \mathcal{K}(s) f(s, u(s)) \mathrm{d} s .
$$

Substituting into (2.7) gives

$$
\lambda u(t)=\frac{\lambda A_{0} \gamma(t)}{\lambda-\Gamma}+\frac{\gamma(t)}{\lambda-\Gamma} \int_{G} \mathcal{K}(s) f(s, u(s)) \mathrm{d} s+\int_{G} k(t, s) f(s, u(s)) \mathrm{d} s .
$$


Taking the absolute value and then the supremum for $t \in G$ yields

$$
\lambda \rho \leqslant \frac{\lambda A_{0}\|\gamma\|}{\lambda-\Gamma}+\frac{\|\gamma\|}{\lambda-\Gamma} \int_{G} \mathcal{K}(s) f(s, u(s)) \mathrm{d} s+\sup _{t \in G} \int_{G}|k(t, s)| f(s, u(s)) \mathrm{d} s .
$$

Thus, since $\lambda>1$, we have

$$
\rho<\frac{A_{0}\|\gamma\|}{1-\Gamma}+\frac{\|\gamma\|}{1-\Gamma} \int_{G} \mathcal{K}(s) \rho \phi_{\rho}(s) \mathrm{d} s+\sup _{t \in G} \int_{G}|k(t, s)| \rho \phi_{\rho}(s) \mathrm{d} s .
$$

This contradicts (2.6) and proves the result.

A simpler variant of Lemma 2.6 is the following.

Lemma 2.7. If the hypothesis (2.6) in Lemma 2.6 is replaced by

$$
\frac{A_{0}\|\gamma\|}{\rho}+A_{1}\|\gamma\|+\sup _{t \in G} \int_{G}|k(t, s)| \phi_{\rho}(s) \mathrm{d} s \leqslant 1,
$$

then the same conclusion holds, that is, $i_{K}\left(T, K_{\rho}\right)=1$.

Proof. Use the inequality $\alpha[u] \leqslant A_{0}+A_{1}\|u\|$ in (2.7).

Remark 2.8. In (2.10) it is implicit that $A_{0}\|\gamma\| / \rho+A_{1}\|\gamma\|<1$.

Remark 2.9. When $G=[0,1], f(s, u)=g(s) h(u)$ with $g$ measurable, $h$ continuous and $\Phi g \in L^{1}(0,1)$, we have $\phi_{\rho}(s)=g(s) h^{-\rho, \rho}$, where

$$
h^{-\rho, \rho}=\sup \left\{\frac{h(u)}{\rho}: u \in[-\rho, \rho]\right\} .
$$

Equation (2.6) then reads more simply:

$$
\frac{A_{0}\|\gamma\|}{\rho(1-\Gamma)}+\left(\frac{\|\gamma\|}{1-\Gamma} \int_{0}^{1} \mathcal{K}(s) g(s) \mathrm{d} s+\frac{1}{m}\right) h^{-\rho, \rho} \leqslant 1
$$

where

$$
\frac{1}{m}=\sup _{t \in[0,1]} \int_{0}^{1}|k(t, s)| g(s) \mathrm{d} s .
$$

This reduces to a result in $[\mathbf{7}]$ when $\alpha[u]=0$.

Later in the paper we shed light on conditions (2.10) and (2.11).

The above results allow us to give the following new result on existence of multiple non-zero solutions for equation (2.1).

Theorem 2.10. Equation (2.1) has a non-zero solution in $K$ if either of the following conditions hold.

$\left(\mathrm{H}_{1}\right)$ There exist $\rho_{1}, \rho_{2} \in(0, \infty)$ with $\rho_{1}<\rho_{2}$ such that $\left(\mathrm{I}_{\rho_{1}}^{1}\right)$ and $\left(\mathrm{I}_{\rho_{2}}^{0}\right)$ hold, and $u \neq T u$ for $u \in \partial V_{\rho_{2}}$. 
$\left(\mathrm{H}_{2}\right)$ There exist $\rho_{1}, \rho_{2} \in(0, \infty)$ with $\rho_{1}<c \rho_{2}$ such that $\left(\mathrm{I}_{\rho_{1}}^{0}\right)$ and $\left(\mathrm{I}_{\rho_{2}}^{1}\right)$ hold, and $u \neq T u$ for $u \in \partial K_{\rho_{2}}$.

Equation (2.1) has two non-zero solutions in $K$ if one of the following conditions holds.

$\left(\mathrm{S}_{1}\right)$ There exist $\rho_{1}, \rho_{2}, \rho_{3}$ with $\rho_{1}<\rho_{2}$ and $\rho_{2}<c \rho_{3}$ such that $\left(\mathrm{I}_{\rho_{1}}^{1}\right)$, $\left(\mathrm{I}_{\rho_{2}}^{0}\right)$ and $\left(\mathrm{I}_{\rho_{3}}^{1}\right)$ hold, and $u \neq T u$ for $u \in \partial V_{\rho_{2}}$.

$\left(\mathrm{S}_{2}\right)$ There exist $\rho_{1}, \rho_{2}, \rho_{3}$ with $\rho_{1}<c \rho_{2}<c \rho_{3}$ such that $\left(\mathrm{I}_{\rho_{1}}^{0}\right),\left(\mathrm{I}_{\rho_{2}}^{1}\right)$ and $\left(\mathrm{I}_{\rho_{3}}^{0}\right)$ hold, and $u \neq T u$ for $u \in \partial K_{\rho_{2}}$.

Moreover, if, in $\left(S_{1}\right)$, strict inequality holds in $\left(\mathrm{I}_{\rho_{1}}^{1}\right)$, then equation (2.1) has a third solution, $u_{0} \in K_{\rho_{1}}$ (possibly zero).

We omit the proof, as it follows simply from properties of the fixed-point index. For details of similar proofs see $[\mathbf{7}, \mathbf{1 2}]$.

Remark 2.11. It is possible to state results for three or more non-zero solutions by expanding the lists in conditions $\left(S_{1}\right)$ and $\left(S_{2}\right)$. We leave these to the reader, who may consult $[\mathbf{1 3}]$ to see the type of result that may be stated.

We now present a version of Theorem 2.10 for the existence of one solution that may be checked more easily than the original.

Theorem 2.12. Let $f(t, u)=g(t) h(u)$ be as above and suppose that

$$
\int_{a}^{b} \Phi(s) g(s) \mathrm{d} s>0 .
$$

Equation (2.1) then has a non-zero solution in $K$ if one of the following conditions holds.

$\left(\mathrm{H}_{1}^{\prime}\right)$ There exist $\rho_{1}, \rho_{2} \in(0, \infty)$ with $\rho_{1}<\rho_{2}$ such that $h^{-\rho_{1}, \rho_{1}}$ satisfies (2.11) and $h_{\rho_{2}, \rho_{2} / c}$ satisfies $\left(\mathrm{I}_{\rho_{2}}^{0}\right)$ with $(2.3)$ replaced by $(2.4)$.

$\left(\mathrm{H}_{2}^{\prime}\right)$ There exist $\rho_{1}, \rho_{2} \in(0, \infty)$ with $\rho_{1}<c \rho_{2}$ such that $h_{\rho_{1}, \rho_{1} / c}$ satisfies $\left(\mathrm{I}_{\rho_{1}}^{0}\right)$ with $(2.3)$ replaced by (2.4) and $h^{-\rho_{2}, \rho_{2}}$ satisfies $(2.11)$.

\section{Non-zero solutions of the BVP (1.1), (1.2)}

We now consider the BVP

$$
-u^{\prime \prime}(t)=f(t, u(t)) \quad \text { a.e. on }[0,1]
$$

with boundary conditions

$$
u^{\prime}(0)+\alpha[u]=0, \quad \beta u^{\prime}(1)+u(\eta)=0, \quad \eta \in[0,1]
$$

The solution of $-u^{\prime \prime}=y$ under these BCs can be written as

$$
u(t)=(\beta+\eta-t) \alpha[u]+\beta \int_{0}^{1} y(s) \mathrm{d} s+\int_{0}^{\eta}(\eta-s) y(s) \mathrm{d} s-\int_{0}^{t}(t-s) y(s) \mathrm{d} s .
$$


By a solution of the BVP (3.1), (3.2) we mean a solution $u \in C[0,1]$ of the corresponding integral equation

$$
u(t)=(\beta+\eta-t) \alpha[u]+\int_{0}^{1} k(t, s) f(s, u(s)) \mathrm{d} s,
$$

where

$$
k(t, s)=\beta+(\eta-s) H(\eta-s)-(t-s) H(t-s),
$$

and

$$
H(x)= \begin{cases}1, & x \geqslant 0, \\ 0, & x<0 .\end{cases}
$$

Note that $k(t, s)$ in $(3.3)$ is the kernel for the special case $u^{\prime}(0)=0$ studied in [9]. Here we discuss the case $\beta>0$ and $\beta+\eta<1$. When $\beta+\eta \geqslant 1$, similar calculations lead to the existence of positive solutions.

\subsection{Upper bounds}

Note that

$$
\|\gamma\|= \begin{cases}\beta+\eta, & \text { for } \beta+\eta \geqslant \frac{1}{2}, \\ 1-(\beta+\eta), & \text { for } \beta+\eta<\frac{1}{2} .\end{cases}
$$

In [9] it was shown that, when $\beta>0$ and $\beta+\eta<1, k(t, s)$ changes sign and one may take

$$
\Phi(s)= \begin{cases}\beta+\eta, & \text { for } \beta+\eta \geqslant \frac{1}{2}, \\ 1-(\beta+\eta), & \text { for } \beta+\eta<\frac{1}{2} .\end{cases}
$$

\subsection{Lower bounds}

We take $[0, b]$ with $\eta<b<\eta+\beta$. Note that, in $[\eta, b], \gamma(t)$ is a decreasing function of $t$ and

$$
\min _{t \in[0, b]} \gamma(t)=\beta+\eta-b
$$

A simple calculation shows that $k(t, s) \geqslant \beta+\eta-b$ for $t \in[0, b]$. This leads to

$$
c= \begin{cases}\frac{\beta+\eta-b}{\beta+\eta}, & \text { for } \beta+\eta \geqslant \frac{1}{2}, \\ \frac{\beta+\eta-b}{1-(\beta+\eta)}, & \text { for } \beta+\eta<\frac{1}{2} .\end{cases}
$$

Hence we work on the cone

$$
K=\left\{u \in C[0,1], \min _{t \in[0, b]} u(t) \geqslant c\|u\|\right\},
$$

with $c$ as in (3.4). 
Remark 3.1. For the four-point BVP corresponding to $\alpha[u]=\alpha u(\xi)$ with $0 \leqslant \xi \leqslant b$, $\alpha>0$, note that $\alpha[u] \geqslant 0$ for $u \in K$. We may take $A_{0}=0$, and $\mathrm{d} A(s)$ to be the Dirac measure of weight $\alpha$ at $\xi$. Then

$$
\Gamma=\int_{0}^{1} \gamma(t) \mathrm{d} A(t)=\alpha \gamma(\xi)=\alpha(\beta+\eta-\xi)
$$

and $\mathcal{K}(s)=\alpha k(\xi, s)$. For the case $f(s, u)=g(s) h(u), i_{K}\left(T, K_{\rho}\right)=1$ if $\Gamma<1$ and

$$
\left[\frac{\alpha\|\gamma\|}{1-\Gamma} \int_{0}^{1} k(\xi, s) g(s) \mathrm{d} s+\sup _{t \in[0,1]} \int_{0}^{1}|k(t, s)| g(s) \mathrm{d} s\right] h^{-\rho, \rho} \leqslant 1 .
$$

Alternatively, we may use (2.10). Then $i_{K}\left(T, K_{\rho}\right)=1$ if

$$
\alpha\|\gamma\|+\left[\sup _{t \in[0,1]} \int_{0}^{1}|k(t, s)| g(s) \mathrm{d} s\right] h^{-\rho, \rho} \leqslant 1 .
$$

Of course (3.6) can only be valid if $\alpha\|\gamma\|<1$, which is more stringent than $\Gamma<1$ in the first part. Equation (3.6) is simpler to apply but (3.5) can be less restrictive on $f$.

Since $0 \leqslant k(\xi, s) \leqslant \max _{[0,1]}|k(t, s)|,(3.5)$ is satisfied if

$$
\left(\frac{\alpha\|\gamma\|}{1-\Gamma}+1\right) h^{-\rho, \rho} \leqslant m
$$

In particular, when $\xi=0$ and $\beta+\eta \geqslant \frac{1}{2}$, so that $\|\gamma\|=\gamma(0)=\beta+\eta$, we get (3.6).

Example 3.2. Consider the BVP

$$
-u^{\prime \prime}(t)=h(u(t)) \quad \text { a.e. on }[0,1]
$$

with boundary conditions

$$
u^{\prime}(0)+\alpha u(\xi)=0, \quad \beta u^{\prime}(1)+u(\eta)=0, \quad 0 \leqslant \eta \leqslant 1, \quad 0 \leqslant \xi \leqslant b<\beta+\eta .
$$

As above, $i_{K}\left(T, K_{\rho}\right)=1$ if

$$
\left[\frac{\alpha\|\gamma\|}{1-\alpha \gamma(\xi)} \int_{0}^{1} k(\xi, s) \mathrm{d} s+\sup _{t \in[0,1]} \int_{0}^{1}|k(t, s)| \mathrm{d} s\right] h^{-\rho, \rho} \leqslant 1 .
$$

We calculate

$$
\sup _{t \in[0,1]} \int_{0}^{1}|k(t, s)| \mathrm{d} s .
$$

For $t \leqslant \beta+\eta, k(t, s) \geqslant 0$ for every $t$ and $s$, so

$$
\int_{0}^{1}|k(t, s)| \mathrm{d} s=\int_{0}^{1} \beta \mathrm{d} s+\int_{0}^{\eta}(\eta-s) \mathrm{d} s-\int_{0}^{t}(t-s) \mathrm{d} s=\beta+\frac{1}{2} \eta^{2}-\frac{1}{2} t^{2}
$$

and the maximum occurs at $t=0$. 
For $t>\beta+\eta, k(t, s)$ changes sign, so

$$
\begin{aligned}
\int_{0}^{1}|k(t, s)| \mathrm{d} s & \\
\quad= & \int_{0}^{\eta}[t-(\beta+\eta)] \mathrm{d} s+\int_{\eta}^{t-\beta}(t-s-\beta) \mathrm{d} s+\int_{t-\beta}^{t} \beta-(t-s) \mathrm{d} s+\int_{t}^{1} \beta \mathrm{d} s \\
= & {[t-(\beta+\eta)] \eta+\frac{1}{2}\left(t-(\beta+\eta)^{2}\right)+\frac{1}{2} \beta^{2}+(1-t) \beta . }
\end{aligned}
$$

This quadratic has its maximum at one of the endpoints, that is either when $t=\beta+\eta$ or when $t=1$. Hence, the maximum on $[0,1]$ is for either $t=0$ or $t=1$, and thus

$$
\sup _{t \in[0,1]} \int_{0}^{1}|k(t, s)| \mathrm{d} s=\max \left\{\beta+\frac{1}{2} \eta^{2}, \beta^{2}-\beta+\frac{1}{2}\left(1-\eta^{2}\right)\right\} \text {. }
$$

The maximum is $\beta+\frac{1}{2} \eta^{2}$ if and only if $\beta \geqslant 1-\left(\frac{1}{2}+\eta^{2}\right)^{1 / 2}$. From the equation above we obtain

$$
\int_{0}^{1} k(\xi, s) \mathrm{d} s=\beta+\frac{1}{2} \eta^{2}-\frac{1}{2} \xi^{2} .
$$

Hence, all numbers in (3.9) can be calculated.

We calculate some numbers with which to compare (3.9) with the simpler expression that arises from (3.6), namely

$$
\alpha\|\gamma\|+\left[\sup _{t \in[0,1]} \int_{0}^{1}|k(t, s)| \mathrm{d} s\right] h^{-\rho, \rho} \leqslant 1 .
$$

Take $\eta=\xi=\frac{1}{2}, \alpha=\beta=\frac{1}{4},\|\gamma\|=\beta+\eta=\frac{3}{4}$. Then $\beta \geqslant 1-\left(\frac{1}{2}+\eta^{2}\right)^{1 / 2}$ so

$$
\sup _{t \in[0,1]} \int_{0}^{1}|k(t, s)| \mathrm{d} s=\beta+\frac{1}{2} \eta^{2}=\frac{3}{8} \text {. }
$$

Formula (3.10) requires $\frac{3}{8}+\frac{3}{8} h^{-\rho, \rho} \leqslant 1$, or $h^{-\rho, \rho} \leqslant \frac{5}{3}$, and (3.9) requires

$$
\left[\frac{3}{8}\left(1-\frac{1}{4}\left(\frac{1}{4}\right)\right)^{-1} \cdot \frac{1}{4}+\frac{3}{8}\right] h^{-\rho, \rho} \leqslant 1 \quad \text { or } h^{-\rho, \rho} \leqslant \frac{40}{19} .
$$

This shows that, for this case, (3.6) is weaker than (3.5).

We state a result for the existence of one non-trivial solution when $f(t, u)=g(t) h(u)$, for which the hypotheses are easier to check. Of course there are more general results, including existence of multiple non-zero solutions, analogous to Theorem 2.10.

Theorem 3.3. Let $[a, b]=[0, b]$, with $\eta<b<\eta+\beta$, where $\eta+\beta<1$, and suppose that

$$
\int_{0}^{b} \Phi(s) g(s) \mathrm{d} s>0 .
$$

Let $c$ be as in (3.4). Let $m$ be as in (2.12) and $M$ as in (2.5). Then the BVP (3.1), (3.2) has at least one non-zero solution, positive on $[0, b]$, if either $\left(\mathrm{H}_{1}^{\prime}\right)$ or $\left(\mathrm{H}_{2}^{\prime}\right)$ of Theorem 2.12 hold. 
Remark 3.4. These techniques allow us to discuss positivity of solutions as $\beta$ changes. We have given above the details for case (ii) below. The other cases are treated with similar methods; we omit the details of these cases.

(i) When $\beta \geqslant 1-\eta$, we have $\gamma(t) \geqslant 0$ and $k(t, s) \geqslant 0$. Thus, $T u(t) \geqslant 0$ and, with the same methods, we can prove the existence of positive solutions on all of $[0,1]$.

(ii) When $0<\beta<1-\eta$, both $\gamma(t)$ and $k(t, s)$ change sign and we have shown the existence of solutions that are positive on an interval $[0, b]$.

(iii) When $\beta<0$ and $\beta+\eta>0, k(t, s)$ is negative for $t \in[\beta+\eta, 1]$. This case can be dealt with by considering $v(t)=-u(t)$ as in $[\mathbf{8}]$. We achieve the existence of solutions that are negative on an interval $[a, 1]$ for $\beta+\eta<a<1$.

(iv) When $\beta<0$ and $\beta+\eta<0, k(t, s)$ is negative for all $t$ and $s$ and we may show, as in case (iii), the existence of negative solutions on all of $[0,1]$.

\section{Non-zero solutions of the BVP (1.1)-(1.3)}

We now consider the BVP

$$
-u^{\prime \prime}(t)=f(t, u(t)) \quad \text { a.e. on }[0,1]
$$

with boundary conditions

$$
u(0)=\alpha[u] \geqslant 0, \quad \beta u^{\prime}(1)+u(\eta)=0, \quad \eta \in[0,1] .
$$

The solution of $-u^{\prime \prime}=y$ under these BCs is

$u(t)=\left(1-\frac{t}{\beta+\eta}\right) \alpha[u]+\frac{\beta t}{\beta+\eta} \int_{0}^{1} y(s) \mathrm{d} s+\frac{t}{\beta+\eta} \int_{0}^{\eta}(\eta-s) y(s) \mathrm{d} s-\int_{0}^{t}(t-s) y(s) \mathrm{d} s$.

By a solution of the BVP (4.1), (4.2) we shall mean a solution $u \in C[0,1]$ of the corresponding integral equation

$$
u(t)=\left(1-\frac{t}{\beta+\eta}\right) \alpha[u]+\int_{0}^{1} k(t, s) f(s, u(s)) \mathrm{d} s,
$$

where

$$
k(t, s)=\frac{\beta t}{\beta+\eta}+\frac{t}{\beta+\eta}(\eta-s) H(\eta-s)-(t-s) H(\eta-s) .
$$

When $\beta \geqslant 0$,

$$
k(t, s) \text { and }\left(1-\frac{t}{\beta+\eta}\right) \alpha[u]
$$

both change sign when $0<\beta+\eta<1$, but are positive on the strip $0 \leqslant t \leqslant b, b<\beta+\eta$. This is the case for which we give details and apply the results of $\S 2$, taking $[a, b]=[\eta, b]$ for a suitable $b$ with $\eta<b<\beta+\eta$. 


\subsection{Upper bounds}

First note that

$$
\|\gamma\|= \begin{cases}1, & \text { for } \beta+\eta \geqslant \frac{1}{2} \\ {\left[\frac{1-(\beta+\eta)}{\beta+\eta}\right],} & \text { for } \beta+\eta<\frac{1}{2} .\end{cases}
$$

Second, we are looking for $\Phi(s)$ such that

$$
|k(t, s)| \leqslant \Phi(s) .
$$

If $s \leqslant \eta$ and $s \leqslant t$,

$$
k(t, s)=\frac{s(\beta+\eta-t)}{\beta+\eta}
$$

is decreasing in $t$, so $\left|k_{\max }\right|$ is either $k(s, s)$ or $-k(1, s)$. Therefore,

$$
k(t, s) \leqslant \max \left\{\frac{s(\beta+\eta-s)}{\beta+\eta}, \frac{s[1-(\beta+\eta)]}{\beta+\eta}\right\} .
$$

We omit the calculations for the other cases.

The result is that we may take (for simplicity)

$$
\Phi(s)= \begin{cases}s, & \text { for } \beta+\eta \geqslant \frac{1}{2}, \\ {\left[\frac{1-(\beta+\eta)}{\beta+\eta}\right] s,} & \text { for } \beta+\eta<\frac{1}{2} .\end{cases}
$$

\subsection{Lower bounds}

We take $[\eta, b]$ with $\eta<b<\eta+\beta$.

Note that in $[\eta, b], \gamma(t)$ is a decreasing function of $t$ and

$$
\min _{t \in[\eta, b]} \gamma(t)=\frac{\beta+\eta-b}{\beta+\eta} .
$$

If $\beta+\eta \geqslant \frac{1}{2}, \Phi(s)=s$ and we check that we may choose

$$
c=\min \left\{\frac{\beta \eta}{\beta+\eta}, \frac{\beta+\eta-b}{\beta+\eta}\right\} .
$$

If $\beta+\eta<\frac{1}{2}$,

$$
\Phi(s)=\left[\frac{1-(\beta+\eta)}{\beta+\eta}\right] s,
$$

and, for example, when $s \leqslant \eta$ and $s \leqslant t$,

$$
k(t, s)=\frac{s(\beta+\eta-t)}{\beta+\eta} \geqslant \frac{s(\beta+\eta-b)}{\beta+\eta},
$$


so we need

$$
c \leqslant \frac{\beta+\eta-b}{1-(\beta+\eta)} .
$$

Other regions are treated similarly. Hence, we obtain

$$
c=\min \left\{\frac{\beta \eta}{1-(\beta+\eta)}, \frac{\beta+\eta-b}{1-(\beta+\eta)}\right\}
$$

for $\beta+\eta<\frac{1}{2}$.

Theorem 4.1. Let $[a, b]=[\eta, b]$, with $\eta<b<\eta+\beta$, where $\beta+\eta \geqslant \frac{1}{2}$, and suppose that

$$
\int_{0}^{b} \Phi(s) g(s) \mathrm{d} s>0 .
$$

Let $c$ be as in (4.4). Let $m$ be as in (2.12) and $M$ as in (2.5). Then the BVP (4.1), (4.2) has at least one non-zero solution, positive on $[0, b]$, if either $\left(\mathrm{H}_{1}^{\prime}\right)$ or $\left(\mathrm{H}_{2}^{\prime}\right)$ of Theorem 2.12 holds.

An analogous result holds for $\beta+\eta<\frac{1}{2}$ but with $c$ as in (4.5).

Remark 4.2. The ranges of $\beta$ and the type of solutions obtained are the same as in Remark 3.4, with the exception of the case when $\beta<0$ and $\beta+\eta<0$. In fact, for these particular ranges, for $\mathrm{BC}(1.2)$ there are solutions that are negative on $[0,1]$ but, for $\mathrm{BC}$ (1.3), it turns out that again there exist positive solutions; this is because $\beta+\eta=0$ is a singular case for this BVP. In fact, when $\beta<0$ and $\beta+\eta<0$, a routine calculation shows that $k(t, s) \geqslant 0$.

\section{Positivity results}

We study a positivity problem for the BVP

$$
-u^{\prime \prime}(t)=f(t, u(t))
$$

with a special case of the type of boundary condition we are studying, namely

$$
u^{\prime}(0)+\alpha u(\xi)=0, \quad \beta u^{\prime}(1)+u(\eta)=0,
$$

where $0 \leqslant \alpha<1$ and $0 \leqslant \xi \leqslant \beta+\eta<1$.

In [9] we showed a regain-of-positivity result for the special case $u^{\prime}(0)=0$ that was motivated by previous work of Guidotti and Merino [4].

When $0<\beta<1-\eta$ there cannot exist positive solutions for all positive right-hand sides. Nevertheless, under additional conditions on the nonlinearity $f$, we can achieve positive solutions when $\beta$ is not far from $1-\eta$ (with an explicit estimate). Some examples given below show that our estimate is sharp.

We make the following assumptions on the nonlinearity:

(f1) $0 \leqslant f_{\min } \leqslant f(s, u) \leqslant f_{\max }$ for $s \in[0,1], u \in \mathbb{R}$, where $f_{\max }>0$;

(f2) for $0 \leqslant s \leqslant \frac{1}{2}$ and $u \geqslant v, f(s, u) \leqslant f(1-s, v)$. 
For example, (f2) holds if $f(s, u)=g(s) h(u)$, where $g$ is increasing and $h$ is decreasing.

Theorem 5.1. Under assumptions (f1) and (f2), any solution of (5.1), (5.2) is positive on all of $[0,1]$ for

$$
\beta\left(1-\frac{\alpha|\gamma(1)|}{1-\alpha \gamma(\xi)}\right) \geqslant \frac{1}{2}\left[1-\left(1-\frac{\alpha|\gamma(1)|}{1-\alpha \gamma(\xi)}\right) \eta^{2} \frac{f_{\min }}{f_{\max }}-\frac{\alpha|\gamma(1)|}{1-\alpha \gamma(\xi)} \xi^{2} \frac{f_{\min }}{f_{\max }}\right] .
$$

Proof. If $u$ is a solution of the BVP, $u$ satisfies

$$
u(t)=\gamma(t) \alpha u(\xi)+\int_{0}^{1} k(t, s) f(s, u(s)) \mathrm{d} s:=T u(t) .
$$

Since $T u(t)$ is a decreasing function of $t$, a sign-changing solution is positive on $[0,1]$ if and only if $u(1) \geqslant 0$. First, setting $t=\xi$ and, second, setting $t=1$ gives

$$
\begin{aligned}
u(1)=\frac{\alpha \gamma(1)}{1-\alpha \gamma(\xi)} & {\left[\int_{0}^{1} \beta f(s, u(s)) \mathrm{d} s+\int_{0}^{\eta}(\eta-s) f(s, u(s)) \mathrm{d} s-\int_{0}^{\xi}(\xi-s) f(s, u(s)) \mathrm{d} s\right] } \\
& +\int_{0}^{1} \beta f(s, u(s)) \mathrm{d} s+\int_{0}^{\eta}(\eta-s) f(s, u(s)) \mathrm{d} s-\int_{0}^{1}(1-s) f(s, u(s)) \mathrm{d} s .
\end{aligned}
$$

Now

$$
\begin{aligned}
\int_{0}^{1}(1-s) f(s, u(s)) \mathrm{d} s=\int_{0}^{1} \frac{1}{2} f(s, & u(s)) \mathrm{d} s \\
& \quad+\int_{0}^{1 / 2}\left(\frac{1}{2}-s\right) f(s, u(s)) \mathrm{d} s+\int_{1 / 2}^{1}\left(\frac{1}{2}-s\right) f(s, u(s)) \mathrm{d} s,
\end{aligned}
$$

by changing variable in the third integral and then using (f2) as in [9] we get

$$
-\int_{0}^{1}(1-s) f(s, u(s)) \mathrm{d} s \geqslant-\int_{0}^{1} \frac{1}{2} f(s, u(s)) \mathrm{d} s .
$$

So

$$
\begin{aligned}
u(1) \geqslant & {\left[\left(1-\frac{\alpha|\gamma(1)|}{1-\alpha \gamma(\xi)}\right) \beta-\frac{1}{2}\right] \int_{0}^{1} f(s, u(s)) \mathrm{d} s } \\
& +\left(1-\frac{\alpha|\gamma(1)|}{1-\alpha \gamma(\xi)}\right) \int_{0}^{\eta}(\eta-s) f(s, u(s)) \mathrm{d} s+\frac{\alpha|\gamma(1)|}{1-\alpha \gamma(\xi)} \int_{0}^{\xi}(\xi-s) f(s, u(s)) \mathrm{d} s .
\end{aligned}
$$

If

$$
\left(1-\frac{\alpha|\gamma(1)|}{1-\alpha \gamma(\xi)}\right) \beta \geqslant \frac{1}{2}
$$

then $u(1) \geqslant 0$. Otherwise, we have

$$
u(1) \geqslant\left[\left(1-\frac{\alpha|\gamma(1)|}{1-\alpha \gamma(\xi)}\right) \beta-\frac{1}{2}\right] f_{\max }+\left(1-\frac{\alpha|\gamma(1)|}{1-\alpha \gamma(\xi)}\right) \frac{1}{2} \eta^{2} f_{\min }+\frac{\alpha|\gamma(1)|}{1-\alpha \gamma(\xi)} \frac{1}{2} \xi^{2} f_{\min } .
$$


Therefore, $u(1) \geqslant 0$ if

$$
\beta\left(1-\frac{\alpha|\gamma(1)|}{1-\alpha \gamma(\xi)}\right) \geqslant \frac{1}{2}-\left(1-\frac{\alpha|\gamma(1)|}{1-\alpha \gamma(\xi)}\right) \frac{1}{2} \eta^{2} \frac{f_{\min }}{f_{\max }}-\frac{\alpha|\gamma(1)|}{1-\alpha \gamma(\xi)} \frac{1}{2} \xi^{2} \frac{f_{\min }}{f_{\max }}
$$

Remark 5.2. When $\alpha=0$, we obtain the conditions of [9, Theorem 4.1]. When $\xi=0,(5.3)$ reads

$$
\beta\left(1-\frac{\alpha|\gamma(1)|}{1-\alpha \gamma(0)}\right) \geqslant \frac{1}{2}\left[1-\left(1-\frac{\alpha|\gamma(1)|}{1-\alpha \gamma(0)}\right) \eta^{2} \frac{f_{\min }}{f_{\max }}\right] .
$$

Thus,

$$
\beta \geqslant \frac{1}{2}\left[\frac{1-\alpha \gamma(0)}{1-\alpha(\gamma(0)+|\gamma(1)|)}-\eta^{2} \frac{f_{\min }}{f_{\max }}\right] .
$$

Since $\gamma(t)=\beta+\eta-t$, we obtain

$$
\beta \geqslant \frac{1}{2}\left[\frac{1-\alpha(\beta+\eta)}{1-\alpha}-\eta^{2} \frac{f_{\min }}{f_{\max }}\right]
$$

so the explicit form for $\beta$ is given by

$$
\beta \geqslant \frac{1-\alpha \eta}{2-\alpha}-\frac{(1-\alpha)}{2-\alpha} \eta^{2} \frac{f_{\min }}{f_{\max }} .
$$

When $\xi=\eta,(5.3)$ can be written

$$
\beta\left(1-\frac{\alpha|\gamma(1)|}{1-\alpha \gamma(\eta)}\right) \geqslant \frac{1}{2}\left(1-\eta^{2} \frac{f_{\min }}{f_{\max }}\right),
$$

so

$$
\beta\left(\frac{1-\alpha(\gamma(\eta)+|\gamma(1)|)}{1-\alpha \gamma(\eta)}\right) \geqslant \frac{1}{2}\left(1-\eta^{2} \frac{f_{\min }}{f_{\max }}\right) .
$$

Since $\gamma(t)=\beta+\eta-t$, we get

$$
\beta\left(\frac{1-\alpha(1-\eta)}{1-\alpha \beta}\right) \geqslant \frac{1}{2}\left(1-\eta^{2} \frac{f_{\min }}{f_{\max }}\right)
$$

which gives the explicit form

$$
\beta\left(1-\alpha\left(\frac{1}{2}-\eta+\frac{\eta^{2}}{2} \frac{f_{\min }}{f_{\max }}\right)\right) \geqslant \frac{1}{2}\left(1-\eta^{2} \frac{f_{\min }}{f_{\max }}\right) .
$$

The following simple example shows that the estimate on $\beta$ is sharp. 
Example 5.3. Take

$$
-u^{\prime \prime}(t)=2 \quad \text { with BCs } u^{\prime}(0)+\alpha u(\xi)=0, \beta u^{\prime}(1)+u(\eta)=0,
$$

where $0<\alpha<1, \xi \in[0, \eta]$.

The solution is $u(t)=A+B t-t^{2}$, where

$$
\begin{aligned}
B+\alpha\left(A+B \xi-\xi^{2}\right) & =0, \\
\beta(B-2)+\left(A+B \eta-\eta^{2}\right) & =0 .
\end{aligned}
$$

The solution changes sign at

$$
t_{1}:=\frac{1}{2} B+\left(\frac{1}{4} B^{2}+A\right)^{1 / 2},
$$

and so it is positive on all of $[0,1]$ if and only if $A+B \geqslant 1$.

When $\xi=0, B+\alpha A=0$ and $\beta(-\alpha A-2)+\left(A-\alpha A \eta-\eta^{2}\right)=0$. Thus, $A+B \geqslant 1$ if

$$
\frac{2 \beta+\eta^{2}}{1-\alpha(\beta+\eta)}(1-\alpha) \geqslant 1
$$

Hence,

$$
\beta \geqslant \frac{1-\alpha \eta}{2-\alpha}+\frac{1-\alpha}{2-\alpha} \eta^{2}
$$

This agrees with (5.7) and shows that (5.7) is optimal.

When $\xi=\eta, B+\alpha\left(A-\alpha A \eta-\eta^{2}\right)=0$ and $\beta(B-2)+\left(A-\alpha A \eta-\eta^{2}\right)=0$. Thus,

$$
B=-\frac{2 \alpha \beta}{1-\alpha \beta} \quad \text { and } \quad A=\eta^{2}+\frac{2 \beta(1+\alpha \eta)}{1-\alpha \beta} .
$$

The condition $A+B \geqslant 1$ leads to

$$
\beta\left(1-\alpha\left(\frac{1}{2}-\eta+\frac{1}{2} \eta^{2}\right)\right) \geqslant \frac{1}{2}\left(1-\eta^{2}\right) .
$$

This agrees with (5.9), so the estimate is sharp.

\section{Uniqueness results}

We prove some uniqueness results under the familiar assumption that $f$ is a decreasing function of $u$, in the special case when the parameter $\beta$ is positive and the boundary condition at 0 is particularly simple.

Theorem 6.1. Suppose that $f(t, u)$ is continuous and strictly decreasing in $u$ for every fixed $t$. Then, for constants $U \in \mathbb{R}, p \in \mathbb{R}$ and $\eta \in[0,1]$, the equation

$$
-u^{\prime \prime}=f(t, u(t)), \quad t \in(0,1)
$$


subject to one of the following BCs,

(i) $u(0)=U, \beta u^{\prime}(1)+u(\eta)=0, \beta>0$,

(ii) $u^{\prime}(0)+p=0, \beta u^{\prime}(1)+u(\eta)=0, \beta>0$,

has at most one solution.

Proof. (i) Suppose $u$ and $v$ are two solutions and let $w=u-v$. Then

$$
w^{\prime \prime}(t)=f(t, v(t))-f(t, u(t))
$$

so that, for $t \in(0,1)$,

$$
w(t)>0 \Longrightarrow w^{\prime \prime}(t)>0 \text { and } w(t)<0 \Longrightarrow w^{\prime \prime}(t)<0,
$$

that is, $w$ is convex (respectively, concave) when $w>0$ (respectively, $w<0$ ). Also, $w$ satisfies the BCs

$$
w(0)=0, \quad \beta w^{\prime}(1)+w(\eta)=0 .
$$

If $w$ is not identically zero then it must have either a positive maximum or a negative minimum on $[0,1]$. These cannot occur in $(0,1)$ since at such a local extremum we contradict (6.1)

If $w$ has a positive maximum at 1 , then we have $w(t) \geqslant 0$ on $[0,1]$ and so $w$ is convex. Also $w(1)>0$ and $w^{\prime}(1) \geqslant 0$. From the BC in $(6.2)$ we obtain $w(\eta) \leqslant 0$, and hence $w(\eta)=0$ and $w^{\prime}(1)=0$. By convexity we have $w(t) \equiv 0$ on $[0, \eta]$, and hence $w^{\prime}(\eta)=0$. By convexity once more, this yields constant $w(t)$ for $t \in[\eta, 1]$. Hence, $w(t) \equiv 0$ on $[0,1]$ in this case.

The case when $w$ has a negative minimum at 1 is proved similarly.

(ii) Suppose that $u$ and $v$ are two solutions and let $w=u-v$. Then (6.1) holds and $w$ satisfies the $\mathrm{BCs}$

$$
w^{\prime}(0)=0, \quad \beta w^{\prime}(1)+w(\eta)=0 .
$$

As in case (i), $w$ cannot have a positive local maximum or negative local minimum.

First, consider the case $w(0)>0$. Then $w$ is convex and increasing on $[0,1]$. The maximum of $w$ then occurs at 1 and we have $w^{\prime}(1) \geqslant 0$, which implies that $w(\eta) \leqslant 0$ : a contradiction.

The case $w(0)<0$ is treated similarly.

The final case, $w(0)=0$, is treated in (i).

Acknowledgements. This work was partly supported by Fondi PRIN (ex40\%).

\section{References}

1. R. P. AGARWAL And D. O'REGAN, Upper and lower solutions for singular problems with nonlinear boundary data. Nonlin. Diff. Eqns Applic. 9 (2002), 419-440.

2. H. Amann, Fixed point equations and nonlinear eigenvalue problems in ordered Banach spaces, SIAM Rev. 18 (1976), 620-709. 
3. J. V. BAXLEY, A singular nonlinear boundary value problem: membrane response of a spherical cap. SIAM J. Appl. Math. 48 (1988), 497-505.

4. P. Guidotti And S. Merino, Gradual loss of positivity and hidden invariant cones in a scalar heat equation, Diff. Integ. Eqns 13 (2000), 1551-1568.

5. D. Guo And V. LAKShmikantham, Nonlinear problems in abstract cones (Academic, 1988).

6. Ch. P. Gupta, S. K. Ntouyas and P. Ch. Tsamatos, Existence results for multipoint boundary value problems for second order ordinary differential equations, Bull. Greek Math. Soc. 43 (2000), 105-123.

7. G. Infante And J. R. L. WeBb, Nonzero solutions of Hammerstein integral equations with discontinuous kernels, J. Math. Analysis Applic. 272 (2002), 30-42.

8. G. Infante AND J. R. L. WeBB, Three point boundary value problems with solutions that change sign, J. Integ. Eqns Applic. 15 (2003), 37-57.

9. G. Infante And J. R. L. WeBb, Loss of positivity in a nonlinear scalar heat equation, Nonlin. Diff. Eqns Applic., in press (doi:10.1007/s00030-005-0039-y).

10. G. L. Karakostas and P. Ch. Tsamatos, Existence of multiple positive solutions for a nonlocal boundary value problem, Topolog. Meth. Nonlin. Analysis 19 (2002), 109-121.

11. M. A. KRASnosel'skil AND P. P. ZABREIKKO, Geometrical methods of nonlinear analysis (Springer, 1984).

12. K. Q. LAN, Multiple positive solutions of Hammerstein integral equations with singularities, Diff. Eqns Dynam. Syst. 8 (2000), 175-192.

13. K. Q. LAN, Multiple positive solutions of semilinear differential equations with singularities, J. Lond. Math. Soc. 63 (2001), 690-704.

14. K. Q. LAN AND J. R. L. WEBB, Positive solutions of semilinear differential equations with singularities, J. Diff. Eqns 148 (1998), 407-421.

15. R. MA AND N. CASTANEDA, Existence of solutions of nonlinear $m$-point boundary value problems, J. Math. Analysis Applic. 256 (2001), 556-567.

16. R. H. MARTIN, Nonlinear operators and differential equations in Banach spaces (Wiley, 1976).

17. D. O'REGAN, Upper and lower solutions for singular problems arising in the theory of membrane response of a spherical cap, Nonlin. Analysis 47 (2001), 1163-1174.

18. D. O'REGAN, Upper and lower solutions for problems with singular sign changing nonlinearities and with nonlinear boundary data, Topolog. Meth. Nonlin. Analysis 19 (2002), 375-390.

19. J. R. L. WEBB, Positive solutions of some three point boundary value problems via fixed-point-index theory, Nonlin. Analysis 47 (2001), 4319-4332. 